\title{
Wheat genotypes as assessed by Additive main \& multiplicative interactions (AMMI) and Best linear unbiased prediction (BLUP) for stability analysis under rainfed timely sown trials in Northern Hills Zone of India
}

\author{
Ajay Verma* \\ ICAR-Indian Institute of Wheat \& Barley Research, Karnal-132001 (Haryana), India \\ G. P. Singh \\ ICAR-Indian Institute of Wheat \& Barley Research, Karnal-132001 (Haryana), India \\ ${ }^{*}$ Corresponding author. Email: verma.dwr@gmail.com
}

\author{
Article Info \\ https://doi.org/10.31018/ \\ jans.v13i1.2534 \\ Received: February 2, 2021 \\ Revised: March 3, 2021 \\ Accepted: March 5, 2021
}

\section{How to Cite}

Verma, A. and Singh, G.P. (2021). Wheat genotypes as assessed by Additive main \& multiplicative interactions (AMMI) and Best linear unbiased prediction (BLUP) for stability analysis under rainfed timely sown trials in Northern Hills Zone of India. Journal of Applied and Natural Science, 13(1): 220 - 229. https://doi.org/10.31018/jans.v13i1.2534

\begin{abstract}
Stability analysis of wheat genotypes under rainfed timely sown trials in Northern Hills Zone of India by Additive main \& multiplicative interactions (AMMI) analysis observed highly significant effects of the environment, GxE interaction and genotypes during 2018-19 and 2019-20. The ranking of genotypes had altered with utilization of more number of IPCA's in AMMI and WAASB measures. Environments contributed about 53\%, GxE interaction accounted for $30.5 \%$ and Genotypes explained only $5.4 \%$ of the total sum of squares due to treatments in the first year. Wheat genotypes HS668, VL2035, VL2036, HS562 had been selected by Analytic measures of adaptability and Superiority indexes. Different quadrants comprised of a cluster of arithmetic, geometric, harmonic means along with corresponding adaptability measures. Superiority Indexes considering averages grouped separately. This group maintained the right angles with a group of MASV \& MASV1 measures. Clustering of Adaptability measures as per arithmetic, geometric and harmonic means placed in a quadrant. Second-year reflected VL2041, HS675, HS676 \& HS562, HPW471 genotypes selected by adaptability and superiority indexes. About $68 \%$ of the total variation with $38.4 \%$ and $30.2 \%$ contributions by PC1 \& PC2. Adaptability measures maintained the right angle with other stability measures, with the exception of Superiority indexes. There is an additional advantage with these measures to assign variable weights to the yield and stability as per the goal of breeding trials. These indexes have the potential to provide reliable estimates of genotypes in future studies as they are considered more number of significant IPCA's in biplots.
\end{abstract}

Keywords: AMMI model, Biplot analysis, MASV, SI, WAASB

\section{INTRODUCTION}

Large number of stability analysis measures have been developed to model GxE interactions efficiently (Mohammadi et al., 2015; Bocianowski et al., 2019; Agahi et al., 2020). AMMl based stability measures evaluate the stability of genotypes after reduction of the noise from the GxE interaction effects (Gauch 2013; Oyekunle et al., 2017; Ajay et al., 2020). Researchers have introduced various selection criteria for the simultaneous utilization of yield and stability of evaluated genotypes as stable varieties are generally not highly productive ((Kang, 1993; Rao and Prabhakaran 2005; Farshadfar, 2008; Farshadfar et al., 2011). BLUP and AMMI approach utilized to distinguish the useful pattern from the residual noise in interactions analysis procedures (Piepho et al., 2008; Mendas et al., 2012). The benefits of two important techniques AMMI and BLUP amalgamated into a Superiority Index measure for stability and adaptability of genotypes (Olivoto et al., 2019). The present study had assessed the stability and adaptability behaviour of wheat genotypes as per AMMI and BLUP of genotypes evaluated under rainfed timely sown trials in the Northern Hills Zone of India during 2018-19 and 2019-20.

\section{MATERIALS AND METHODS}

Northern hills zone comprised of J\&K (except Jammu and Kathua distt.), Himachal Pradesh (except Una and 
Paonta Valley), Uttarakhand (except Tarai area), Sikkim, hills of West Bengal and North-Eastern states. Sixteen advanced wheat genotypes at eight locations and sixteen genotypes at nine locations were evaluated under field trials at the northern hills zone during 201819 and 2019-20 cropping seasons. Field trials were conducted at research centers of All India Coordinated Wheat \& Barley Improvement Program (AICWBP) in randomized complete block designs with three replications. Recommended agronomic practices were followed to harvest good yield. Details of locations along with parentage of evaluated wheat genotypes are mentioned in Tables 1 and 2 for ready reference.

Stability measure as a Weighted Average of Absolute Scores calculated as

WAASB $=\sum_{k=1}^{p}\left|I P C A_{i k} \times E P_{k}\right| / \sum_{k=1}^{p} E P_{k}$

$W{ }^{\prime} A B_{i}$ was the weighted average of absolute scores of the ith genotype (or environment); $\mathrm{IPCA}_{i k}$ the score of the ith genotype (or environment) in the $k$ th IPCA, and $E_{k}$ was the amount of the variance explained by the $k$ th IPCA. Superiority index allowed variable weights to yield and stability measure (WAASB) to select genotypes that combine high performance and stability as

$$
\mathrm{SI}=\frac{\left(r G_{i} \times \theta_{Y}\right)+\left(r W_{i} \times \theta_{S}\right)}{\left(\theta_{Y}+\theta_{S}\right)}
$$

where $r G_{i}$ and $r W_{i}$ were the rescaled values for yield and WAASB, respectively, for the ith genotype; $G_{i}$ and $W_{i}$ were the yield and the WAASB values for ith genotype. SI superiority index for the ith genotype that weighted between yield and stability, and $\theta Y$ and $\theta S$ were the weights for yield and stability assumed to be

Mohamadi and Amri (2008)

Zali et al., (2012)

Ajay et al. ( 2019)

Resende and Durate (2007)

Resende and Durate (2007)

Oliveto et al. ( 2019)
Geometric Adaptability Index

Modified AMMI stability Value

MASV1

Relative performance of genotypic values across environments

Harmonic mean of Relative performance of genotypic values

Superiority Index of order 65 and 35 respectively in this study,

AMMISOFT version 1.0, available at https://scs.c als.cornell.edu/people/ hugh-gauch/ utilized for AMMI analysis of wheat genotypes evaluated under multilocation trials in the Peninsular Zone and further analysis was carried out by SAS software version 9.3. Stability measures had been compared with recent analytic measures of adaptability calculated as the relative performance of genetic values (PRVG) and harmonic mean based measure of the relative performance of the genotypic values (MHPRVG) for the simultaneous analysis of stability, adaptability, and yield (Mendes et al., 2012).

\section{RESULTS AND DISCUSSION}

\section{First-year (2018-19)}

The present study observed that Environment (E), GxE interaction, and genotypes $(G)$ effects were highly significant $(P>0.001)$ for wheat genotypes evaluated under multi-location trials as mentioned by the AMMI analysis. Analysis observed the greater contribution of environments, GxE interactions, and genotypes to the total sum of squares (SS) compared to the residual effects. Further SS attributable to GXE interactions was partitioned as attributed to GxE interactions Signal and GxE interactions Noise. AMMI analysis is appropriate for data sets where-in SS due to were of magnitude at least of due to additive genotype main effects (Gauch, 2013). The SS for GxE interactions Signal was higher than genotype main effects, indicating the appropriateness of AMMI analysis. The environment significantly explained about 53\%, GxE interaction accounted for

$\mathrm{GAI}=\sqrt[n]{\prod_{\mathrm{k}=1}^{\mathrm{n}} \overline{\mathrm{X}}_{\mathrm{k}}}$ Eq.1

$M A S V=\sqrt{\sum_{n=1}^{N-1} \frac{\operatorname{SSIPC}_{n}}{\operatorname{SSIPC}_{n+1}}\left(P C_{n}\right)^{2}+\left(P C_{n+1}\right)^{2}}$ ...Eq.2

MASV1 $=\sqrt{\sum_{n=1}^{N-1}\left(\frac{S S I P C_{n}}{S S I P C_{n+1}} P C_{n}\right)^{2}+\left(P C_{n+1}\right)^{2}}$

$P R V G_{i j}=V G_{i j} / V G_{i}$

MHPRVG $_{\mathrm{i}}=$ Number of environments $/ \sum_{j=1}^{k} \frac{1}{P R V G_{i j}}$

$\mathrm{SI}=\frac{\left(r G_{i} \times \theta_{Y}\right)+\left(r W_{i} \times \theta_{S}\right)}{\left(\theta_{Y}+\theta_{S}\right)}$ 
Verma, A. and Singh, G.P. / J. Appl. \& Nat. Sci. 13(1): 220 - 229 (2021)

Table 1. Details of locations and parentage of evaluated wheat genotypes 2018-19.

\begin{tabular}{|c|c|c|c|c|c|c|}
\hline Code & $\begin{array}{l}\text { Geno- } \\
\text { type }\end{array}$ & Parentage & Locations & Latitude & Longitude & $\begin{array}{l}\text { Mean sea } \\
\text { level }\end{array}$ \\
\hline G 1 & $\begin{array}{l}\text { HPW } \\
462\end{array}$ & (VL804/PBW498) & Dhaulakuan & $28^{\circ} 59 \mathrm{~N}$ & $77^{\circ} 16 \mathrm{E}$ & 468 \\
\hline G 2 & $\begin{array}{l}\text { HPW } \\
466\end{array}$ & (PASTOR/HXL7573/2*BAU/3/SOKOLL/WBLL1) & Shimla & $31^{\circ} 10^{\prime} \mathrm{N}$ & $77^{\circ} 17^{\prime} \mathrm{E}$ & 2276 \\
\hline G 3 & VL 2038 & (CHINA84-400022/PBW599) & Malan & $32^{\circ} 08^{\prime} \mathrm{N}$ & $76^{\circ} 35^{\prime} \mathrm{E}$ & 846 \\
\hline G 4 & VL 2037 & (HS485/RAJ4174//HS485-5) & Bajaura & $31^{\circ} 50^{\prime} \mathrm{N}$ & $77^{\circ} 9^{\prime} \mathrm{E}$ & 1103.85 \\
\hline G 5 & HS 507 & (KAUZ/MYNA/VUL//BUC/FLK/4/MILAN) & Wadura & $21^{\circ} 18^{\prime} \mathrm{N}$ & $77^{\circ} 41^{\prime} \mathrm{E}$ & 508 \\
\hline G 6 & UP 3038 & (AKAW4510/AVOCET) & Khudwani & $33^{\circ} 70^{\prime} \mathrm{N}$ & $75^{\circ} 10^{\prime} \mathrm{E}$ & 1590 \\
\hline G 7 & VL 2035 & $\begin{array}{l}\text { (PRL/2*PASTOR//PBW343*2/KUKUNA/3/ROLF07/4/ } \\
\text { BERKUT//PBW343*2/KUKUNA }\end{array}$ & Almora & $29^{\circ} 35^{\prime} \mathrm{N}$ & $79^{\circ} 39^{\prime} \mathrm{E}$ & 1610 \\
\hline G 8 & HS 667 & (HPW251/FLW3//HS431) & Ranichauri & $28^{\circ} 43^{\prime} \mathrm{N}$ & $81^{\circ} 02^{\prime} \mathrm{E}$ & 2200 \\
\hline G 9 & HS 668 & (VL906/FLW13) & & & & \\
\hline G 10 & HS 669 & (VL907/VL876) & & & & \\
\hline G 11 & UP 3039 & (HUW640/LBPY06-15(SERI/DUCULA/PBW343) & & & & \\
\hline G 12 & HS 562 & (OASIS/SKUAZ//4*BCN/3/2*PASTOR) & & & & \\
\hline G 13 & VL 2036 & $\begin{array}{l}\text { (SW89.5277/BORL95//SKAUZ/3/PRL/2*PASTOR/4/ } \\
\text { HEILO/5/WHEAR/SOKOLL) }\end{array}$ & & & & \\
\hline G 14 & $\begin{array}{l}\mathrm{HPW} \\
464\end{array}$ & (Raj 3765/WR 251//HW 2045/PBW 493) & & & & \\
\hline G 15 & $\begin{array}{l}\text { HPW } \\
463\end{array}$ & (HPW155/HW4024 (P6) & & & & \\
\hline G 16 & HD 3340 & (DPW621-50/DW1293//DW1285) & & & & \\
\hline
\end{tabular}

$30.5 \%$ and Genotypes explained only $5.4 \%$ of the total sum of squares (Table 3 ). First six significant multiplicative terms explained $98.7 \%$ and the remaining $1.3 \%$ residual was discarded (Oyekunle et al., 2017).

\section{Ranking of genotypes vis-à-vis number of IPCA's}

The stability or adaptability of genotypes over environments had been assessed by their IPCA scores in the AMMI analysis. More the IPCA scores reflected the specific adaptation of genotype to certain locations. Lower the IPCA scores converged to zero, indicating the more stable or adapted genotypes to all the locations. The ranking of genotypes as per the absolute value of IPCA-1 measure were VL2038, HD3340, HPW463, HPW 462 (Table 4). While for IPCA-2, genotypes HPW462, UP3039, VL2036 would be of choice. Values of IPCA-3 favoured HD 3340, HS 562, VL 2038 wheat genotypes. As per IPCA-4, VL 2036, VL 2035, VL 2038 genotypes would be of stable performance. General adaptation of HS507, UP3039, HS667, and specific adaptation of VL2037, VL2038 genotypes pointed by IPCA-5 measure. Genotypes HPW464, HS562, HS667 for the general along with HS669, HS668 for specific locations as identified by absolute values of IPCA-6. Analytic measures of adaptability MASV and MASV1 considered all significant six IPCAs of the analysis. Values of MASV pointed towards genotypes VL2037, HPW466, and HPW463 express stable yield whereas genotypes HPW463, HPW466 \& VL2037 be of stable performance MASV1 measure respectively (Ajay et al 2019).

Change in the ranking of genotypes with the utilization of numbers of significant IPCA's in the WAASB estimation had been also assessed. The genotypes were ranked based on $1,2, \ldots, 6$ IPCA in the WAASB estimation. The genotype with the smallest value of WAASB was ranked with first-order. Genotypes preferences varied as VL2036, UP3039, HS668, VL2037 based on W1 values whereas VL2036, UP3039, VL2035, HS668 as per W2 values while UP 3039, VL2035, VL2037, HS668 by values of W3 (Table 5). Genotypes UP3039, VL2035, VL2036, VL2037 pointed by W4; W5 favoured UP3039, VL2035, VL2036, HS668, and lastly by W6 the genotypes of choice would be UP3039, VL2035, VL2036, HS668. Stability measure WAASB based on all six significant IPCA's settled for UP3039, VL2035, VL2036, HS668 genotypes for considered locations of the zone for stable high yield. The genotypes ranking had been altered by the number of significant IPCAs included in the WAASB calculations. This reinforced the benefits of using the WAASB index since it captured all the variations of IPCAs to compute the stability performance (Olivoto et al., 2019).

\section{Productive and broadly adapted genotypes by AM- MI + BLUP tools}

An average yield of genotypes as per BLUP values selected HS668, HS669, HS562, VL2036 wheat geno- 
Verma, A. and Singh, G.P. / J. Appl. \& Nat. Sci. 13(1): 220 - 229 (2021)

Table 2. Details of locations and parentage of evaluated wheat genotypes 2019-20.

\begin{tabular}{|c|c|c|c|c|c|c|}
\hline Code & Genotype & Parentage & Locations & Latitude & Longitude & $\begin{array}{l}\text { Mean sea } \\
\text { level }\end{array}$ \\
\hline$\overline{\text { G } 1}$ & HS507 & (KAUZ/MYNA/VUL//BUC/FLK/4/MILAN) & Dhaulakuan & $28^{\circ} 59 \mathrm{~N}$ & $77^{\circ} 16 \mathrm{E}$ & 468 \\
\hline G 2 & SKW356 & (SEL-VL968) & Shimla & $31^{\circ} 10^{\prime} \mathrm{N}$ & $77^{\circ} 17^{\prime} \mathrm{E}$ & 2276 \\
\hline G 3 & VL2042 & $\begin{array}{l}\text { (TX71A983.4/TX69D4812//PYN/3/VPM/ } \\
\text { MOS83.11.4-8//PEW/HSB3177 } \\
\text { (Yr15+Yr24)/6*Avocet//2*BAXTER/FLW13 }\end{array}$ & Malan & $32^{\circ} 08^{\prime} \mathrm{N}$ & $76^{\circ} 35^{\prime} \mathrm{E}$ & 846 \\
\hline G 4 & HPW471 & (HPW236/VL900) & Bajaura & $31^{\circ} 50^{\prime} \mathrm{N}$ & $77^{\circ} 9^{\prime} \mathrm{E}$ & 1103.85 \\
\hline G 5 & HS675 & (HS240*2FLW20//HS240*2/FLW13) & Wadura & $21^{\circ} 18^{\prime} \mathrm{N}$ & $77^{\circ} 41^{\prime} \mathrm{E}$ & 508 \\
\hline G 6 & HPW472 & (HPW155/HD29) & Khudwani & $33^{\circ} 70^{\prime} \mathrm{N}$ & $75^{\circ} 10^{\prime} \mathrm{E}$ & 1590 \\
\hline $\begin{array}{l}\text { G } 7 \\
\text { G } 8 \\
\text { G } 9\end{array}$ & $\begin{array}{l}\text { VL2039 } \\
\text { HS677 } \\
\text { HS676 }\end{array}$ & $\begin{array}{l}\text { (RL6043/4*NAC//PASTOR/3/BABAX/VL892) } \\
\text { (ID8900994W/VEE/3/CHEN/AES/HD2932) } \\
\text { (VL907/DL460) }\end{array}$ & $\begin{array}{l}\text { Almora } \\
\text { Ranichauri } \\
\text { Umiam }\end{array}$ & $\begin{array}{l}29^{\circ} 35^{\prime} \mathrm{N} \\
28^{\circ} 43^{\prime} \mathrm{N} \\
25^{\circ} 53^{\prime} \mathrm{N}\end{array}$ & $\begin{array}{l}79^{\circ} 39^{\prime} \mathrm{E} \\
81^{\circ} 02^{\prime} \mathrm{E} \\
91^{\circ} 27^{\prime} \mathrm{E}\end{array}$ & $\begin{array}{l}1610 \\
2200\end{array}$ \\
\hline G 10 & UP3064 & (RAJ3765/HD3121) & & & & \\
\hline G 11 & HS678 & (VL907/HD2997) & & & & \\
\hline G 12 & HS562 & (OASIS/SKUAZ//4*BCN/3/2*PASTOR) & & & & \\
\hline G 13 & HPW470 & (NAC/TH.AC//3*MIRLO/BUC/4PASTOR) & & & & \\
\hline G 14 & HPW469 & (HPW89/VL867) & & & & \\
\hline G 15 & VL2041 & (NESSER/SAULSKU32/MACS6240//HS507) & & & & \\
\hline G 16 & VL2040 & $\begin{array}{l}\text { (17thDSBWYT99(SERI.1B//KAUZ/HEVO/3/ } \\
\text { AMAD/4/PYN/BAU//MILAN/5/ OPATA/ RAY- } \\
\text { ON//KAUZ) }\end{array}$ & & & & \\
\hline
\end{tabular}

Table 3. AMMI analysis of wheat genotypes evaluated under rainfed timely sown trials during 2018-19 \& 2019-20.

\begin{tabular}{|c|c|c|c|c|c|c|}
\hline Source & $\begin{array}{l}\text { Degree of } \\
\text { Freedom } \\
18-19\end{array}$ & $\begin{array}{l}\text { Degree of } \\
\text { Freedom } \\
19-20\end{array}$ & $\begin{array}{l}\text { Mean Sum } \\
\text { of Squares } \\
18-19\end{array}$ & $\begin{array}{l}\text { Mean Sum } \\
\text { of Squares } \\
19-20\end{array}$ & $\begin{array}{l}\text { Level of } \\
\text { Significance } \\
18-19\end{array}$ & $\begin{array}{l}\text { Level of } \\
\text { Significance } \\
19-20\end{array}$ \\
\hline Treatments & 127 & 143 & 242.41 & 218.91 & $.0000000 * * *$ & $.0000000^{* * *}$ \\
\hline Genotypes (G) & 15 & 15 & 124.66 & 185.53 & $.0000000 * * *$ & $.0000000^{* * *}$ \\
\hline $\begin{array}{l}\text { Environments } \\
(\mathrm{E})\end{array}$ & 7 & 8 & 2623.83 & 2167.13 & $.0000000^{* * *}$ & $.0000000 * * *$ \\
\hline $\begin{array}{l}\text { Interactions } \\
\text { GxE }\end{array}$ & 105 & 120 & 100.47 & 93.21 & $.0000000^{* * *}$ & $.0000000^{* * *}$ \\
\hline IPC1 & 21 & 22 & 192.74 & 169.69 & $.0000000^{* * *}$ & $.0000000^{* * *}$ \\
\hline IPC2 & 19 & 20 & 125.04 & 164.26 & $.0000000^{* * *}$ & $.0000000^{* * *}$ \\
\hline IPC3 & 17 & 18 & 108.06 & 104.59 & $.0000000^{* * *}$ & $.0000000^{* * *}$ \\
\hline IPC4 & 15 & 16 & 69.20 & 77.15 & $.0000000^{* * *}$ & $.0000000^{* * *}$ \\
\hline IPC5 & 13 & 14 & 51.74 & 33.00 & $.0000000^{* * *}$ & $.0000050^{* * *}$ \\
\hline \multirow[t]{2}{*}{ IPC6 } & 11 & 12 & 40.62 & 27.24 & $.0000422^{* * *}$ & $.0022316^{* *}$ \\
\hline & & 10 & & 23.61 & & 0.1065 \\
\hline Residual & 9 & 8 & 14.67 & 3.00 & 0.1609 & 0.9657 \\
\hline Error & 384 & 432 & 10.05 & 10.01 & & \\
\hline Total & 511 & 575 & 67.80 & 61.96 & & \\
\hline
\end{tabular}

types is given in Table 6. This measure was simple but not exploited the full information contained in the dataset. Geometric mean was suggested to evaluate the adaptability of genotypes. Geometric mean observed HS668, VL2036, HS562, VL2035 were of top-ranked genotypes. As Resende (2007) proposed, a method to rank genotypes considering the yield and stability, simultaneously is the harmonic mean of genetic values (HMGV). In the context of mixed models, the Harmonic Mean of Genotypic Values were calculated as genotypes with greater values would be recommended. Harmonic Mean of yield expressed higher values for 
Verma, A. and Singh, G.P. / J. Appl. \& Nat. Sci. 13(1): 220 - 229 (2021)

Table 4. Modified AMMI stability values as per significant IPCA's 2018-19.

\begin{tabular}{llllllllllll}
\hline Genotype & IPCA1 & IPCA2 & IPCA3 & IPCA4 & IPCA5 & IPCA6 & MASV1 & MASV & $\mathbf{R}_{\text {IPCA1 }}$ & $\mathbf{R}_{\text {MASV }}$ & $\mathbf{R}_{\text {MASV1 }}$ \\
\hline HPW 462 & -2.027 & 0.249 & 1.608 & -0.684 & -1.015 & 0.401 & 5.281 & 4.271 & 4 & 7 & 5 \\
HPW 466 & -1.355 & -1.601 & -1.116 & -1.940 & 0.477 & -0.302 & 5.558 & 4.763 & 6 & 2 & 2 \\
VL 2038 & 2.506 & 0.744 & 0.332 & -0.261 & -1.566 & 0.645 & 5.368 & 4.360 & 1 & 6 & 4 \\
VL 2037 & 0.385 & 1.165 & 0.460 & -0.918 & 2.521 & 1.083 & 5.450 & 4.815 & 13 & 1 & 3 \\
HS 507 & 1.249 & -2.793 & 0.435 & 0.522 & 0.035 & 0.711 & 5.253 & 4.720 & 8 & 4 & 6 \\
UP 3038 & 0.910 & -1.310 & 1.888 & -1.084 & -0.662 & -0.685 & 5.252 & 4.446 & 11 & 5 & 7 \\
VL 2035 & 0.478 & -0.612 & 0.622 & -0.184 & 0.703 & -0.790 & 2.370 & 2.066 & 12 & 16 & 16 \\
HS 667 & 1.657 & 2.070 & 0.550 & -0.430 & -0.140 & 0.281 & 4.630 & 3.992 & 5 & 9 & 11 \\
HS 668 & -0.333 & 0.979 & 0.960 & 0.720 & 0.931 & -1.338 & 3.614 & 3.197 & 14 & 13 & 14 \\
HS 669 & 1.091 & 0.649 & -1.798 & 0.360 & -0.312 & -1.829 & 4.695 & 3.983 & 10 & 11 & 9 \\
UP 3039 & 0.049 & -0.278 & -0.855 & 0.888 & -0.070 & 0.940 & 2.607 & 2.260 & 15 & 15 & 15 \\
HS 562 & 1.142 & -0.625 & -0.303 & 1.648 & 0.525 & -0.125 & 3.913 & 3.314 & 9 & 12 & 13 \\
VL 2036 & -0.033 & -0.299 & -2.438 & -0.040 & -0.194 & 0.834 & 5.062 & 4.179 & 16 & 8 & 8 \\
HPW 464 & -1.293 & 1.697 & -1.009 & -0.947 & -0.740 & 0.069 & 4.646 & 3.991 & 7 & 10 & 10 \\
HPW 463 & -2.197 & 0.632 & 0.910 & 2.038 & -0.259 & 0.446 & 5.742 & 4.729 & 3 & 3 \\
HD 3340 & -2.229 & -0.667 & -0.248 & 0.313 & -0.234 & -0.341 & 4.060 & 3.187 & 2 & 14 & 12
\end{tabular}

Table 5. Weighted average of absolute scores and ranks of wheat genotypes 2018-19.

\begin{tabular}{lllllllllllllll}
\hline Genotype & W1 & W2 & W3 & W4 & W5 & W6 & WAASB & $\mathbf{R}_{\mathbf{W} 1}$ & $\mathbf{R}_{\mathbf{W} 2}$ & $\mathbf{R}_{\mathbf{W} \mathbf{3}}$ & $\mathbf{R}_{\mathbf{W} 4}$ & $\mathbf{R}_{\mathbf{W} \mathbf{5}}$ & $\mathbf{R}_{\mathbf{W} 6}$ & $\mathbf{R}_{\mathbf{W A A S B}}$ \\
\hline HPW 462 & 2.03 & 1.33 & 1.40 & 1.30 & 1.27 & 1.21 & 1.21 & 13 & 9 & 12 & 12 & 13 & 13 & 13 \\
HPW 466 & 1.35 & 1.45 & 1.37 & 1.45 & 1.35 & 1.28 & 1.28 & 11 & 10 & 11 & 15 & 15 & 15 & 15 \\
VL 2038 & 2.51 & 1.81 & 1.44 & 1.27 & 1.30 & 1.26 & 1.26 & 16 & 14 & 14 & 10 & 14 & 14 & 14 \\
VL 2037 & 0.38 & 0.69 & 0.63 & 0.67 & 0.85 & 0.86 & 0.86 & 4 & 5 & 3 & 4 & 5 & 6 & 6 \\
HS 507 & 1.25 & 1.86 & 1.50 & 1.36 & 1.23 & 1.20 & 1.20 & 9 & 16 & 15 & 14 & 12 & 12 & 12 \\
UP 3038 & 0.91 & 1.07 & 1.28 & 1.25 & 1.19 & 1.16 & 1.16 & 6 & 8 & 9 & 9 & 9 & 10 & 10 \\
VL 2035 & 0.48 & 0.53 & 0.55 & 0.50 & 0.52 & 0.54 & 0.54 & 5 & 3 & 2 & 2 & 2 & 2 & 2 \\
HS 667 & 1.66 & 1.82 & 1.50 & 1.35 & 1.23 & 1.17 & 1.17 & 12 & 15 & 16 & 13 & 11 & 11 & 11 \\
HS 668 & 0.33 & 0.59 & 0.68 & 0.69 & 0.71 & 0.75 & 0.75 & 3 & 4 & 4 & 5 & 4 & 4 & 4 \\
HS 669 & 1.09 & 0.92 & 1.14 & 1.03 & 0.96 & 1.02 & 1.02 & 7 & 6 & 7 & 7 & 7 & 8 & 8 \\
UP 3039 & 0.05 & 0.14 & 0.32 & 0.40 & 0.37 & 0.41 & 0.41 & 2 & 2 & 1 & 1 & 1 & 1 & 1 \\
HS 562 & 1.14 & 0.94 & 0.78 & 0.90 & 0.86 & 0.81 & 0.81 & 8 & 7 & 6 & 6 & 6 & 5 & 5 \\
VL 2036 & 0.03 & 0.14 & 0.72 & 0.63 & 0.59 & 0.60 & 0.60 & 1 & 1 & 5 & 3 & 3 & 3 & 3 \\
HPW 464 & 1.29 & 1.45 & 1.34 & 1.28 & 1.23 & 1.15 & 1.15 & 10 & 11 & 10 & 11 & 10 & 9 & 9 \\
HPW 463 & 2.20 & 1.58 & 1.41 & 1.50 & 1.38 & 1.32 & 1.32 & 14 & 12 & 13 & 16 & 16 & 16 & 16 \\
HD 3340 & 2.23 & 1.61 & 1.27 & 1.13 & 1.05 & 1.00 & 1.00 & 15 & 13 & 8 & 8 & 8 & 7 & 7 \\
\hline
\end{tabular}

$R_{W 1}, R_{W 2}, R_{W 3}, R_{W 4}, R_{W 5}, R_{W 6}, R_{W A A B}=$ Rank of genotypes as per number of IPCA's in WAASB values

HS668, HS562, HS 669, VL2035 genotypes.

Moreover, the Harmonic Mean of Relative Performance of Genotypic Values (HMRPGV) proposed by Resende (2007) was similar to the methods of Lin and Binns (1988) and Annicchiarico (1992). The genotypes could be simultaneously sorted by yield and stability using the harmonic means of the yield and genotypic performance with smaller the standard deviation among the locations would be recommended/identified values of HMRPGV ranked HS668, HS669, HS562, VL2035 the performance of the genotypes among the locations. The relative performance of genetic values (RPGV) overcrop years was recommended while simultaneously considering the yield and adaptability. The relative performance of Genotypic Values had settled for HS668, HS669, HS562, VL2036 wheat genotypes.

While assigning 65 and 35 weights to yield and stability, the Superiority index pointed out that HS 668, HS669, HS562, VL2035 genotypes would maintain high yield and stable performance. SI considered GAI 
Verma, A. and Singh, G.P. / J. Appl. \& Nat. Sci. 13(1): 220 - 229 (2021)

Table 6. Superiority index and analytic adaptability measures based on BLUP's of genotypes 2018-19.

\begin{tabular}{lllllllllllllllll}
\hline Genotype AMu & Rk & SI au & Rk & GMu & Rk & SI gu & Rk & HMu & Rk & SI hu & Rk & RPGVu Rk & MHRPGVu & Rk \\
\hline HPW 462 & 29.82 & 14 & 18.91 & 14 & 29.09 & 14 & 19.82 & 14 & 28.35 & 13 & 22.29 & 13 & 0.940 & 14 & 0.914 & 14 \\
HPW 466 & 31.34 & 10 & 32.87 & 11 & 30.14 & 11 & 27.50 & 11 & 28.89 & 11 & 24.22 & 12 & 0.975 & 11 & 0.946 & 11 \\
VL 2038 & 33.21 & 5 & 54.45 & 8 & 31.88 & 7 & 45.71 & 8 & 30.50 & 9 & 39.13 & 9 & 1.031 & 7 & 1.004 & 7 \\
VL 2037 & 33.17 & 6 & 69.11 & 7 & 32.39 & 6 & 65.82 & 7 & 31.68 & 5 & 64.33 & 6 & 1.041 & 5 & 1.025 & 6 \\
HS 507 & 30.83 & 13 & 30.53 & 12 & 29.48 & 13 & 24.17 & 13 & 27.78 & 14 & 17.90 & 14 & 0.961 & 13 & 0.918 & 13 \\
UP 3038 & 31.91 & 9 & 43.95 & 9 & 31.29 & 9 & 43.61 & 9 & 30.67 & 7 & 44.32 & 8 & 1.007 & 8 & 0.988 & 9 \\
VL 2035 & 32.87 & 7 & 78.32 & 4 & 32.47 & 4 & 79.12 & 2 & 32.08 & 4 & 80.26 & 2 & 1.038 & 6 & 1.033 & 4 \\
HS 667 & 31.09 & 11 & 34.56 & 10 & 29.96 & 12 & 30.10 & 10 & 28.75 & 12 & 27.37 & 11 & 0.970 & 12 & 0.940 & 12 \\
HS 668 & 34.38 & 1 & 86.70 & 1 & 34.08 & 1 & 86.70 & 1 & 33.78 & 1 & 86.70 & 1 & 1.094 & 1 & 1.080 & 1 \\
HS 669 & 34.07 & 2 & 72.85 & 6 & 33.20 & 2 & 67.67 & 6 & 32.28 & 3 & 63.39 & 7 & 1.065 & 2 & 1.052 & 2 \\
UP 3039 & 32.34 & 8 & 77.57 & 5 & 31.49 & 8 & 74.46 & 5 & 30.60 & 8 & 72.59 & 4 & 1.007 & 9 & 1.001 & 8 \\
HS 562 & 33.85 & 3 & 78.53 & 3 & 33.17 & 3 & 75.46 & 4 & 32.43 & 2 & 72.81 & 3 & 1.064 & 3 & 1.051 & 3 \\
VL 2036 & 33.78 & 4 & 85.87 & 2 & 32.46 & 5 & 76.58 & 3 & 30.99 & 6 & 68.47 & 5 & 1.044 & 4 & 1.025 & 5 \\
HPW 464 & 28.77 & 15 & 9.69 & 16 & 27.53 & 15 & 6.76 & 16 & 26.23 & 16 & 6.32 & 16 & 0.892 & 15 & 0.862 & 15 \\
HPW 463 & 30.88 & 12 & 26.53 & 13 & 30.21 & 10 & 26.83 & 12 & 29.52 & 10 & 28.29 & 10 & 0.979 & 10 & 0.947 & 10 \\
HD 3340 & 28.47 & 16 & 12.20 & 15 & 27.49 & 16 & 12.20 & 15 & 26.43 & 15 & 13.92 & 15 & 0.891 & 16 & 0.858 & 16 \\
\hline
\end{tabular}

$\mathrm{AMu}, \mathrm{GMu}, \mathrm{HMu}=$ Arithmetic, Geometric, Harmonic Mean for BLUP values; SI au, SI gu, SI hu = Superiority index as per Arithmetic, Geometric, Harmonic Mean; RPGVu, MHRPGVu = Relative performance and Harmonic mean of Relative Performance as per BLUP of genotypes; Rk = Rank of genotypes

Table 7. Loadings of measures as per two principal components 2018-19.

\begin{tabular}{lcl}
\hline Measure & PC1 & PC2 \\
\hline IPCA1 & 0.0972 & -0.2907 \\
IPCA2 & 0.0083 & -0.0480 \\
IPCA3 & -0.0633 & -0.1197 \\
IPCA4 & 0.0783 & 0.0153 \\
IPCA5 & 0.1381 & 0.0057 \\
IPCA6 & -0.0538 & 0.1050 \\
MASV1 & -0.1728 & -0.3107 \\
MASV & -0.1607 & -0.3240 \\
W1 & -0.2212 & -0.1059 \\
W2 & -0.2429 & -0.1347 \\
W3 & -0.2524 & -0.1737 \\
W4 & -0.2512 & -0.1861 \\
W5 & -0.2488 & -0.2209 \\
W6 & -0.2422 & -0.2496 \\
WAASB & -0.2422 & -0.2496 \\
AMu & 0.2277 & -0.2968 \\
SI au & 0.2653 & -0.1191 \\
GMu & 0.2355 & -0.2784 \\
SI gu & 0.2692 & -0.0993 \\
HMu & 0.2367 & -0.2542 \\
SI hu & 0.2696 & -0.0769 \\
RPGVu & 0.2284 & -0.3010 \\
MHRPGVu & 0.2403 & -0.2603 \\
\% variance & 57.83 & 14.33 \\
explained & & \\
\hline
\end{tabular}

and stability selected HS668, VL2035, VL2036, HS562 genotypes. The SI while using HM and stability favoured the same set of wheat genotypes HS668, VL2035, HS 562, UP 3039. Analytic adaptability measures RPGV and MHRPGV pointed out HS668, HS 669, HS562, VL2036, HS668, HS669, HS562, VL2035 genotypes would be more adaptable.

\section{Biplot analysis of measures}

The Principal components analysis was performed to study the relationships among the stability and adaptability measures based on BLUP of wheat genotypes. Loadings of the considered measures were tabulated as per the first two significant PC as explained $72.2 \%$ of the total variation in the original variables with respective contributions were $57.8 \& 14.3$ percent (Table 7 ). Four groups of measures were observed in graphical Biplot analysis. The smallest group comprised of MASV \& MASV1 measures (Fig. 1). The nearby group clustered stability measures by utilizing the varying number of the interaction of principal components for calculation. Adaptability measures as per arithmetic, geometric and harmonic means and their corresponding values expressed bondage in a group placed in a different quadrant. However, this group maintained the right angle with stability measures. This quadrant also showed a cluster of Superiority indexes as per averages of the yield of wheat genotypes. Performance of genotypes would be different by Superiority indexes as compared to their behaviour as per values of MASV \& MASV1 measures.

\section{Second-year of study 2019-20}

The AMMI analysis of variance of 16 wheat genotypes evaluated over nine locations revealed that $7.8 \%$ of the total sum of squares (SS) was attributable to the genotypes (G), $48.6 \%$ to the environments (E), and $3.4 \%$ to GxE interaction effects (Table 3). Large SS due to $E$ indicated that the locations were quite diverse induced 


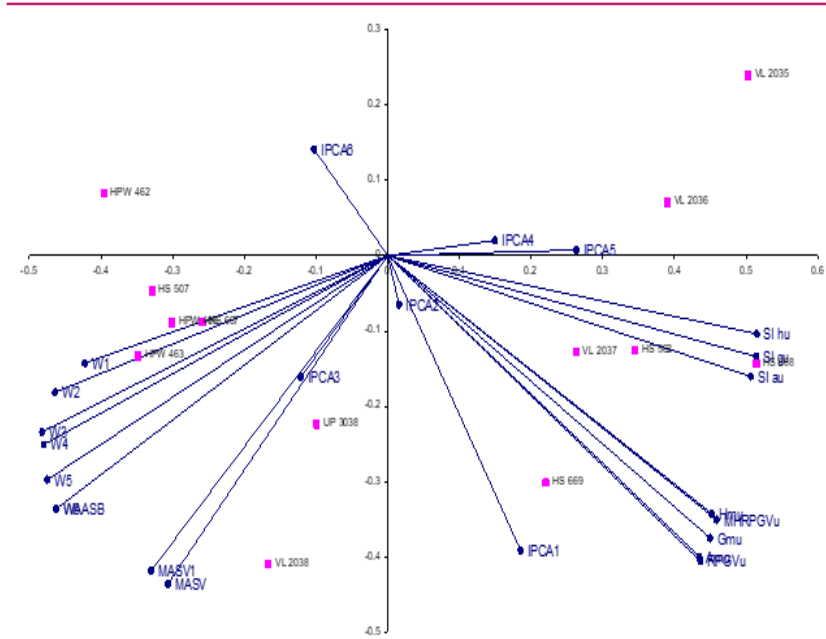

Fig. 1. Biplot analysis of stability \& adaptability measures of wheat genotypes 2018-19.

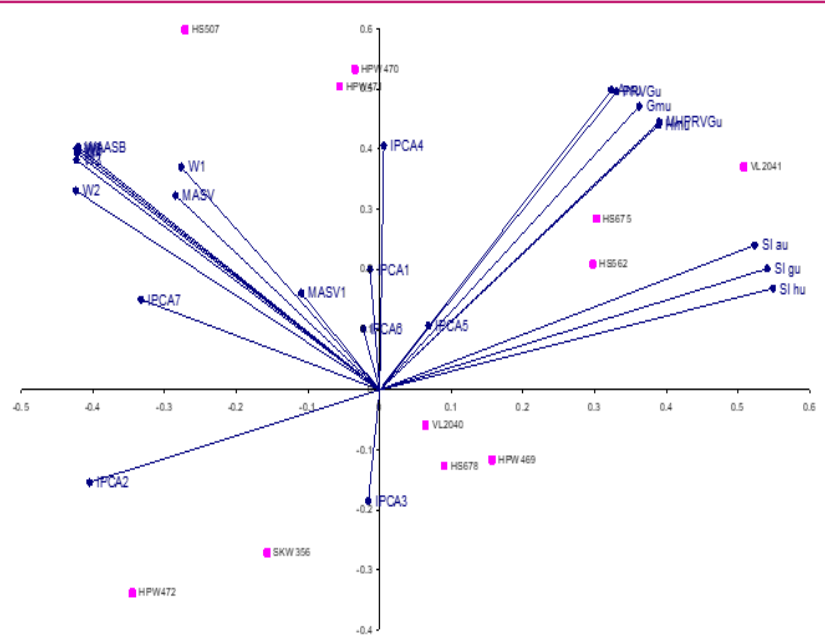

Fig. 2. Biplot analysis of stability \& adaptability measures of wheat genotypes 2019-20.

Table 8. Modified AMMI stability values as per significant IPCA's 2019-20.

\begin{tabular}{lllllllllllll}
\hline Genotype & IPCA1 & IPCA2 & IPCA3 & IPCA4 & IPCA5 & IPCA6 & IPCA7 & MASV1 & MASV & $R_{\text {IPCA1 }}$ & $\mathbf{R}_{\text {MASV1 }}$ & $\mathbf{R}_{\text {MASV }}$ \\
\hline HS507 & -2.617 & 0.220 & -1.903 & 2.230 & -0.350 & 0.362 & 0.074 & 8.862 & 6.252 & 16 & 12 & 15 \\
SKW356 & -2.146 & 0.293 & -0.027 & -0.335 & -1.198 & 0.327 & 1.019 & 5.506 & 3.761 & 13 & 3 & 3 \\
VL2042 & 2.456 & 1.972 & -0.708 & 0.150 & -0.648 & 0.649 & 0.342 & 8.826 & 5.284 & 14 & 11 & 12 \\
HPW471 & 2.549 & -1.057 & -0.752 & -0.242 & -0.279 & 0.330 & 0.899 & 6.321 & 4.307 & 15 & 5 & 6 \\
HS675 & 0.897 & 1.471 & -0.936 & 1.054 & 0.177 & -0.042 & -1.278 & 4.691 & 3.807 & 11 & 2 & 4 \\
HPW472 & 0.496 & 2.214 & -0.506 & -0.407 & 1.209 & 0.278 & 0.105 & 5.607 & 4.299 & 4 & 4 & 5 \\
VL2039 & -0.849 & 2.483 & 1.391 & -0.664 & 0.306 & -0.880 & 0.621 & 10.770 & 5.830 & 10 & 13 & 14 \\
HS677 & -0.717 & 0.183 & -0.327 & -2.283 & -0.316 & -0.642 & -0.357 & 7.664 & 4.334 & 6 & 8 & 7 \\
HS676 & -0.291 & -0.459 & 0.113 & 0.464 & -0.308 & -0.856 & -0.686 & 8.597 & 3.142 & 3 & 10 & 2 \\
UP3064 & 0.078 & -1.003 & 0.511 & -0.371 & -0.977 & -0.204 & -1.057 & 3.725 & 2.772 & 2 & 1 & 1 \\
HS678 & -0.795 & 0.327 & 2.574 & 0.414 & 0.097 & 0.349 & -0.362 & 8.290 & 5.252 & 8 & 9 & 11 \\
HS562 & 0.832 & -1.061 & 1.520 & 0.298 & 0.193 & 1.130 & -0.711 & 12.247 & 5.212 & 9 & 14 & 10 \\
HPW470 & 1.394 & -1.342 & 0.993 & 1.663 & -0.160 & -1.547 & 0.943 & 16.202 & 6.715 & 12 & 16 & 16 \\
HPW469 & 0.062 & -1.382 & -1.634 & -1.358 & -0.478 & -0.335 & -0.259 & 6.730 & 4.573 & 1 & 6 & 8 \\
VL2041 & -0.629 & -1.503 & -0.831 & -0.137 & 2.346 & -0.393 & 0.101 & 6.746 & 4.877 & 5 & 7 & 9 \\
VL2040 & -0.720 & -1.356 & 0.522 & -0.476 & 0.385 & 1.474 & 0.606 & 14.943 & 5.598 & 7 & 15 & 13 \\
\hline
\end{tabular}

differences among the yields of genotypes. The small proportion of SS due to $\mathrm{G}$ indicated that the differences among the genotypes were not very high. The magnitude of GxE SS was 4.38 times more than that for the SS due to $G$, thus, indicating that the differences in the response of the genotypes across environments were substantial and the genotypes need multi-locational testing. The first seven significant multiplicative terms explained $99.9 \%$ of interactions sum of squares.

Ranking of genotypes vis-à-vis number of IPCA's Wheat genotypes HPW469, UP3064, HS676, HPW472 were ranked as per absolute IPCA-1 scores for general adaptation (Table 8). In contrast, IPCA-2 selected, HS677, HS507, SKW356 genotypes as of choice for considered locations of the zone. Values of IPCA-3 favoured SKW356, HS676, HS677 wheat genotypes. As per IPCA-4, VL2041, VL2042, HPW471 genotypes would be of stable performance. HS678, HPW470, HS675 genotypes pointed by IPCA-5 measure for general and VL2041, HPW472 for specific locations. Genotypes for general adaptation were HS675, UP3064, HPW472 identified by absolute values of IPCA-6 and HPW469, VL2040 for specific locations. Lastly, IPCA-7 
Verma, A. and Singh, G.P. / J. Appl. \& Nat. Sci. 13(1): 220 - 229 (2021)

Table 9. Weighted average of absolute scores and ranks of genotypes 2019-20.

\begin{tabular}{|c|c|c|c|c|c|c|c|c|c|c|c|c|c|c|}
\hline $\begin{array}{l}\text { Geno- } \\
\text { type }\end{array}$ & W1 & W2 & W3 & W4 & W5 & W6 & WAASB & $\mathbf{R}_{\mathbf{W} 1}$ & $\mathbf{R}_{\mathrm{W} \mathbf{2}}$ & $\mathbf{R}_{\mathrm{W} 3}$ & $\mathbf{R}_{\mathrm{W} 4}$ & $\mathbf{R}_{\mathrm{W} 5}$ & $\mathbf{R}_{\mathrm{W6}}$ & $\mathbf{R}_{\text {WAASB }}$ \\
\hline HS507 & 2.617 & 1.438 & 1.549 & 1.651 & 1.573 & 1.515 & 1.459 & 16 & 13 & 13 & 16 & 16 & 16 & 16 \\
\hline SKW356 & 2.146 & 1.235 & 0.947 & 0.855 & 0.876 & 0.850 & 0.856 & 13 & 10 & 6 & 5 & 5 & 5 & 6 \\
\hline VL2042 & 2.456 & 2.218 & 1.858 & 1.602 & 1.545 & 1.503 & 1.457 & 14 & 16 & 16 & 15 & 15 & 15 & 15 \\
\hline HPW471 & 2.549 & 1.815 & 1.561 & 1.364 & 1.299 & 1.253 & 1.239 & 15 & 15 & 14 & 13 & 13 & 12 & 12 \\
\hline HS675 & 0.897 & 1.179 & 1.121 & 1.111 & 1.055 & 1.007 & 1.018 & 11 & 9 & 10 & 11 & 11 & 11 & 11 \\
\hline HPW472 & 0.496 & 1.341 & 1.142 & 1.032 & 1.043 & 1.007 & 0.971 & 4 & 11 & 11 & 10 & 10 & 10 & 10 \\
\hline VL2039 & 0.849 & 1.653 & 1.591 & 1.452 & 1.383 & 1.359 & 1.330 & 10 & 14 & 15 & 14 & 14 & 14 & 14 \\
\hline HS677 & 0.717 & 0.455 & 0.424 & 0.702 & 0.679 & 0.677 & 0.665 & 6 & 2 & 2 & 3 & 3 & 3 & 3 \\
\hline HS676 & 0.291 & 0.374 & 0.312 & 0.334 & 0.333 & 0.358 & 0.370 & 3 & 1 & 1 & 1 & 1 & 1 & 1 \\
\hline UP3064 & 0.078 & 0.533 & 0.528 & 0.504 & 0.533 & 0.517 & 0.539 & 2 & 3 & 3 & 2 & 2 & 2 & 2 \\
\hline HS678 & 0.795 & 0.565 & 1.044 & 0.950 & 0.898 & 0.872 & 0.852 & 8 & 4 & 8 & 7 & 6 & 6 & 5 \\
\hline HS562 & 0.832 & 0.945 & 1.082 & 0.965 & 0.918 & 0.928 & 0.920 & 9 & 6 & 9 & 8 & 7 & 7 & 9 \\
\hline HPW470 & 1.394 & 1.369 & 1.279 & 1.336 & 1.266 & 1.279 & 1.266 & 12 & 12 & 12 & 12 & 12 & 13 & 13 \\
\hline HPW469 & 0.062 & 0.711 & 0.931 & 0.995 & 0.964 & 0.934 & 0.908 & 1 & 5 & 5 & 9 & 9 & 8 & 8 \\
\hline VL2041 & 0.629 & 1.059 & 1.005 & 0.875 & 0.963 & 0.936 & 0.904 & 5 & 8 & 7 & 6 & 8 & 9 & 7 \\
\hline VL2040 & 0.720 & 1.033 & 0.911 & 0.846 & 0.818 & 0.849 & 0.840 & 7 & 7 & 4 & 4 & 4 & 4 & 4 \\
\hline
\end{tabular}

settled for HS507, VL2041, HPW472 genotypes for the studied locations of the zone. MASV and MASV1consider all significant IPCAs of the analysis. Values of analytic measures of adaptability MASV1 identified genotypes UP3064, HS675, SKW356, HPW472 would express stable yield whereas genotypes UP3064, HS676, SKW356, and HS675 be of stable performance by MASV measure respectively.

Values of W1 measure prefered HPW469, UP3064, HS676, HPW472 genotypes whereas W2 measure settled for HS676, HS677, UP3064, HS678 while genotypes HS676, HS677, UP3064, VL2040 by W3 values (Table 9). Wheat genotypes HS676 UP3064, HS677, VL2040 were pointed by W4; values of W5 measure favoured HS676, UP3064, HS677, VL2040, and lastly by W6 values genotypes of choice would be HS676, UP3064, HS677, VL2040. WAASB stability measure utilized all significant IPCA's settled for HS676, UP3064, HS677, VL2040 genotypes for considered locations of the zone for stable high yield. The genotype ranking was altered by the number of IPCAs included in the WAASB measure.

\section{Productive and broadly adapted genotypes by AM- MI + BLUP tools}

The average of BLUP's values ranked VL2041, HS675, HS507 \& HPW471 genotypes as the order of choice (Table 10). Superiority index selected VL2041, HS675, HS562 \& HS676 genotypes. Higher values of GM associated with VL2041, HS675, HPW470, HPW471 genotypes. Moreover, the superiority index values found the suitability of VL2041, HS675, HS676 \& HS562.

The harmonic mean of yield settled for VL2041, HS675, HPW470 \& HPW471 genotypes. While superiority in- dex while assigning 65 and 35 percent weight to yield and stability found VL2041, HS676, HS675 \& HS562 as recommended genotypes. Measures of adaptability RPGV and MHRPGV measures selected VL2041, HS675, HPW470 \& HPW471 along with the least stable yield of VL2039.

\section{Biplot analysis of measures}

The loadings of the measures were reflected in Table 11 as per the first two significant Principal Components as these PCs accounted for $68.8 \%$ of the total variation in the original variables. Most of the measures had clustered in five major groups by Biplot analysis (Fig 2). The cluster of MASV1 with IPCA1 \& IPCA6 was observed near to origin. Group of stability measures as per a different number of interaction principal components observed near to cluster of MASV \& W1 measure. The cluster of arithmetic, geometric, harmonic means along with corresponding adaptability measures was seen in a different quadrant. Group of Superiority Indexes considering averages were also found in the same quadrant. Clusters of this quadrant maintained right angles with clusters of other quadrants. This group maintained the right angles with a group of MASV \& MASV1 measures. Moreover, different behaviour of genotypes would be expected as adaptability measures in comparison to the performance by stability measures.

\section{Conclusion}

GxE interaction study in multi-environment trials was carried out by a well-established AMMI model. The simultaneous consideration of stability measures and yield 
Verma, A. and Singh, G.P. / J. Appl. \& Nat. Sci. 13(1): 220 - 229 (2021)

Table 10. Superiority index and adaptability measures of genotypes 2019-20.

\begin{tabular}{lllllllllllllllll}
\hline $\begin{array}{l}\text { Geno- } \\
\text { type }\end{array}$ & AMu & Rk & SI au & Rk & GMu & Rk & SI gu & Rk & HMu & Rk & SI hu & Rk & RPGVu & Rk MHRPGVu Rk \\
\hline HS507 & 29.88 & 3 & 57.21 & 10 & 28.47 & 6 & 51.72 & 11 & 26.87 & 6 & 45.59 & 12 & 1.075 & 5 & 1.028 & 6 \\
SKW356 & 26.64 & 13 & 50.60 & 13 & 25.16 & 13 & 46.68 & 13 & 23.42 & 14 & 41.26 & 13 & 0.943 & 13 & 0.911 & 14 \\
VL2042 & 27.01 & 10 & 34.26 & 14 & 25.85 & 12 & 32.45 & 15 & 24.71 & 12 & 30.79 & 15 & 0.974 & 12 & 0.930 & 12 \\
HPW471 & 29.77 & 4 & 63.38 & 6 & 28.86 & 4 & 61.69 & 6 & 27.97 & 4 & 60.24 & 7 & 1.075 & 4 & 1.054 & 4 \\
HS675 & 29.92 & 2 & 71.71 & 2 & 29.38 & 2 & 72.61 & 2 & 28.81 & 2 & 73.13 & 3 & 1.094 & 2 & 1.072 & 2 \\
HPW472 & 24.98 & 15 & 33.56 & 15 & 24.09 & 15 & 35.08 & 14 & 23.22 & 15 & 36.20 & 14 & 0.901 & 15 & 0.874 & 15 \\
VL2039 & 22.75 & 16 & 4.13 & 16 & 21.47 & 16 & 4.13 & 16 & 20.24 & 16 & 4.13 & 16 & 0.812 & 16 & 0.771 & 16 \\
HS677 & 25.92 & 14 & 51.02 & 12 & 25.00 & 14 & 51.64 & 12 & 24.05 & 13 & 51.78 & 11 & 0.929 & 14 & 0.915 & 13 \\
HS676 & 26.98 & 12 & 68.93 & 4 & 26.47 & 10 & 71.97 & 3 & 25.92 & 8 & 74.09 & 2 & 0.977 & 10 & 0.975 & 8 \\
UP3064 & 26.98 & 11 & 63.56 & 5 & 26.34 & 11 & 65.61 & 5 & 25.65 & 10 & 66.85 & 5 & 0.974 & 11 & 0.968 & 11 \\
HS678 & 27.45 & 9 & 57.20 & 11 & 26.62 & 8 & 57.56 & 9 & 25.83 & 9 & 57.97 & 8 & 0.992 & 8 & 0.971 & 10 \\
HS562 & 29.41 & 6 & 70.82 & 3 & 28.71 & 5 & 70.81 & 4 & 27.94 & 5 & 70.32 & 4 & 1.068 & 6 & 1.049 & 5 \\
HPW470 & 29.65 & 5 & 61.59 & 7 & 28.97 & 3 & 61.60 & 7 & 28.30 & 3 & 61.61 & 6 & 1.078 & 3 & 1.058 & 3 \\
HPW469 & 27.75 & 7 & 57.83 & 9 & 26.89 & 7 & 57.79 & 8 & 25.98 & 7 & 57.22 & 9 & 0.998 & 7 & 0.986 & 7 \\
VL2041 & 30.85 & 1 & 82.85 & 1 & 30.26 & 1 & 82.85 & 1 & 29.69 & 1 & 82.85 & 1 & 1.125 & 1 & 1.107 & 1 \\
VL2040 & 27.55 & 8 & 58.46 & 8 & 26.52 & 9 & 57.27 & 10 & 25.45 & 11 & 55.79 & 10 & 0.985 & 9 & 0.971 & 9 \\
\hline
\end{tabular}

Table 11. Loadings of measures as per two principal components 2019-20

\begin{tabular}{lll}
\hline Measure & PC1 & PC2 \\
\hline IPCA1 & -0.0077 & 0.1216 \\
IPCA2 & -0.2322 & -0.0939 \\
IPCA3 & -0.0091 & -0.1125 \\
IPCA4 & 0.0033 & 0.2469 \\
IPCA5 & 0.0390 & 0.0646 \\
IPCA6 & -0.0134 & 0.0616 \\
IPCA7 & -0.1912 & 0.0912 \\
MASV1 & -0.0629 & 0.0976 \\
MASV & -0.1633 & 0.1964 \\
W1 & -0.1591 & 0.2254 \\
W2 & -0.2433 & 0.2015 \\
W3 & -0.2428 & 0.2324 \\
W4 & -0.2426 & 0.2395 \\
W5 & -0.2413 & 0.2421 \\
W6 & -0.2428 & 0.2436 \\
WAASB & -0.2416 & 0.2458 \\
AMu & 0.1854 & 0.3034 \\
SI au & 0.3002 & 0.1463 \\
GMu & 0.2077 & 0.2866 \\
SI gu & 0.3104 & 0.1225 \\
HMu & 0.2234 & 0.2680 \\
SI hu & 0.3150 & 0.1025 \\
RPGVu & 0.1895 & 0.3015 \\
MHRPGVu & 0.2239 & 0.2709 \\
\% variance & 38.36 & 30.42 \\
\hline & & \\
explained & & \\
\hline
\end{tabular}

would be more appropriate to recommend high-yielding stable wheat genotypes. In the present study, the main advantages of AMMI and BLUP were combined to increase the reliability of multi-locations trials analysis. An additional advantage was provided by Superiority Indexes to assign variable weights to the yield and stability performance. Depending upon the goal of crop breeding trials, the researchers may prioritize the productivity of a genotype rather than its stability (and vice-versa). The stability index of genotype performance has the potential to provide reliable estimates of stability in future studies along with a joint interpretation of performance and stability in biplots while considering the number of significant IPCA's.

\section{ACKNOWLEDGEMENTS}

The wheat genotypes were evaluated at research fields at Coordinated centres of AICW\&BIP across the country. The first author sincerely acknowledges the hard work of all the staff for field evaluation and data recording of wheat genotypes.

\section{Conflict of interest}

The authors declare that they have no conflict of interest.

\section{REFERENCES}

1. Agahi, K., Ahmadi, J., Oghan, H. A., Fotokian, M. H. and Orang, S. F. (2020). Analysis of genotype $\times$ environment interaction for seed yield in spring oilseed rape using the AMMI model. Crop Breeding and Applied Biotechnology 
Verma, A. and Singh, G.P. / J. Appl. \& Nat. Sci. 13(1): 220 - 229 (2021)

20(1), e26502012

2. Ajay, B. C., Aravind, J., Fiyaz, R. A., Kumar, N., La, I. C., Kona, P., Dagla, M. C. and Bera, S. K. (2019). Rectification of modified AMMI stability value (MASV). Indian J. Genet., 79(4), 726-731

3. Ajay, B.C., Bera S.K., Singh A.L., Kumar N., Gangadhar K. and Kona, P. (2020). Evaluation of Genotype $\times$ Environment interaction and yield stability analysis in peanut under phosphorus stress condition using stability parameters of AMMI Model. Agric. Res., 9, 477-486

4. Annicchiarico, P. (1992). Cultivar adaptation and recommendation from alfalfa trials in northern Italy. Journal of Genetics and Plant Breeding, 46, 269-278

5. Bocianowski, J., Niemann J and Nowosad, K. (2019). Genotype-by environment interaction for seed quality traits in interspecific cross-derived Brassica lines using additive main effects and multiplicative interaction model. Euphytica, 215(7),1-13

6. Farshadfar, E. (2008). Incorporation of AMMI stability value and grain yield in a single non-parametric index (GSI) in bread wheat. Pak J Biol Science 11:1791-1796

7. Farshadfar, E., Mahmodi N. and Yaghotipoor, A. (2011). AMMI stability value and simultaneous estimation of yield and yield stability in bread wheat (Triticum aestivum L.). Aust J Crop Science, 5,1837-1844

8. Gauch, H.G. (2013). A simple protocol for AMMI analysis of yield trials. Crop Science, 53,1860-1869

9. Kang, M.S. (1993). Simultaneous selection for yield and stability in crop performance trials: Consequences for growers. Agronomy Journal, 85,754-757

10. Lin, C.S. and Binns, M.R. (1988). A superiority measure of cultivar performance for cultivar $\mathrm{x}$ location data. Canadian Journal of Plant Science, 68, 193-198

11. Mendes, F. F., Guimarães L. J. M., Souza J. C., Guimarães, P. E. O., Pacheco C. A. P., Machado, J. R. de A., Meirelles, W. F., Silva A. R. da and Parentoni, S. N. (2012). Adaptability and stability of maize varieties using mixed model methodology. Crop Breeding and Applied

Biotechnology 12(2), 111-117

12. Mohammadi, M, Sharifi, P., Karimizadeh, R., Jafarby, J.A., Khanzadeh, H., Hosseinpour, T., Poursiabidi, M.M., Roustaii, M., Hassanpour, H.M. and Mohammadi, P. (2015). Stability of grain yield of durum wheat genotypes by AMMI model. Agric For., 61(3), 181-193

13. Mohammadi, R. and Amri, A. (2008). Comparison of parametric and non-parametric methods for selecting stable and adapted durum wheat genotypes in variable environments. Euphytica, 159, 419-432

14. Olivoto, T., Lucio, A. Dal'Col, Gonzalez, Silva J.A. da and Marchioro, V.S. (2019). Mean performance and stability in multi-environment trials I: Combining features of AMMI and BLUP techniques. Agronomy Journal, 111,1-12

15. Oyekunle, M., Menkir, A., Mani, H., Olaoye G., Usman, I.S., Ado, S. (2017). Stability analysis of maize cultivars adapted to tropical environments using AMMI analysis. Cereal Res. Commun., 45,336-345

16. Piepho, H.P., Mo"hring J., Melchinger, A.E. and Bu"chse, A. (2008). BLUP for phenotypic selection in plant breeding and variety testing. Euphytica 161(1),209-228

17. Rao, A.R. and Prabhakaran V.T. (2005). Use of AMMI in simultaneous selection of genotypes for yield and stability. Journal of the Indian Society of Agricultural Statistics, $59,76-82$

18. Resende, M.D.V. (2007). Software Selegen - REML/ BLUP: sistema estatístico e seleção genética computadorizada via modelos lineares mistos. Embrapa Florestas, Colombo, 350p.

19. Resende, M.D.V. and Duarte J.B. (2007). Precision and Quality Control in Variety Trials. Pesquisa Agropecuaria Tropical, 37, 182-194

20. Zali, H., Farshadfar E., Sabaghpour S.H. and Karimizadeh R. (2012). Evaluation of genotype $\times$ environment interaction in chickpea using measures of stability from AMMI model. Ann Biol Res., 3,3126-3136 